\title{
Engagement e Burnout na docência no ensino superior na área da saúde: revisão integrativa
}

\author{
Engagement and Burnout syndrome in faculty \\ members in higher education of health area: \\ integrative review
}

\section{Lucas Filadelfo Meyer ${ }^{1}$ (1) \\ Tamires Elisa Gehr ${ }^{2}$ (1) \\ Letícia dos Santos Gonçalves ${ }^{3}$ (])}

\section{Juliana Ollé Mendes ${ }^{4}$ (1) \\ Débora Maria Vargas Makuch ${ }^{5}$ (1) Ivete Palmira Sanson Zagonel ${ }^{6}$ (1)}

${ }^{1}$ Autor para correspondência. Faculdades Pequeno Príncipe (Curitiba). Paraná, Brasil. lucasfmeyer@gmail.com ${ }^{2-6}$ Faculdades Pequeno Príncipe (Curitiba).Paraná, Brasil. tamiresgehr@gmail.com, leticiasgoncalves2016@gmail.com, julianaolle.mendes@gmail.com, deboramakuch@hotmail.com, ivete.zagonel@fpp.edu.br

RESUMO | INTRODUÇÃO: A docência no ensino superior na área da saúde se enquadra como uma categoria profissional intensamente exposta a estressores psicossociais. Por conseguinte, os fatores atrelados ao engagement e à Síndrome de Burnout (SB) são constructos que permeiam o exercício docente. OBJETIVO: Evidenciar pela revisão de literatura científica como se dá o engagement e Síndrome de Burnout na docência no ensino superior na área da saúde. MATERIAIS E MÉTODOS: A busca pelos estudos foi realizada nas bases de dados Biblioteca Virtual em Saúde (BVS), Web of Science e Science Direct; uma vez aplicados os critérios de inclusão e exclusão, resultaram 6 artigos. RESULTADOS: Conforme o ano de publicação, 33,3\% dos artigos foram desenvolvidos em 2017, 33\% em 2013 seguidos por 17\% em 2012 e em 2011 respectivamente; em se tratando dos resultados dos trabalhos selecionados, quanto à SB tem-se com maior frequência as variáveis idade, sexo, estado civil e atividades de extensão do docente; para que haja a prevenção da SB é necessário propiciar um ambiente de trabaIho saudável, em que deve-se observar a amplificação de políticas, procedimentos e sistemas concebidos, de modo a fazer com que os colaboradores se tornem capazes de atingir os objetivos organizacionais e, concomitantemente, alcancem satisfação em sua ocupação profissional. CONCLUSÃO: Desta forma, conclui-se que, a partir de ambientes adequados de trabalho, estímulos ao desenvolvimento docente, relações laborais saudáveis, estrutura propícia à prática docente, e favorecimento da saúde física, mental e psíquica destes profissionais, as instituições perceberão resultados positivos no processo de ensino e aprendizagem.

PALAVRAS-CHAVE: Engagement. Burnout. Ensino superior. Docência. Saúde.
ABSTRACT | INTRODUCTION: Teaching in higher education in the health field fits as a professional category intensely exposed to psychosocial stressors. Therefore, the factors linked to engagement and the Burnout Syndrome are constructs that permeate teaching. OBJECTIVE: To investigate the scientific literature on engagement and Burnout Syndrome in teaching in higher education in the health area. The search for studies was carried out in BVS, Web of Science, and Science Direct databases; once the inclusion and exclusion criteria were applied, 6 articles resulted. RESULTS: According to the year of publication, 33.3\% of the articles were developed in 2017, 33\% in 2013, followed by $17 \%$ in 2012 and 2011, respectively; when it comes to the results of the selected works, as for BS, the variables age, sex, marital status, and teacher extension activities are more frequently; in order for there to be prevention of BS, it is necessary to provide a healthy work environment, where the amplification of policies, procedures, and systems designed must be observed, in order to make employees capable of achieving organizational objectives and, concomitantly, achieve satisfaction in their professional occupation. CONCLUSION: Thus, it is concluded that from adequate work environments, incentives for teacher development, healthy labor relations, a favorable structure for teaching practice, and favoring the physical, mental and psychological health of these professionals, the institutions will perceive positive results in the process of teaching and learning.

KEYWORDS: Engagement. Burnout. Higher education. Faculty. Health. 


\section{Introdução}

O ensino superior tem passado por intensas modificações no processo ensino-aprendizagem, fato significativamente notório no ensino na área da saúde. A necessidade do desenvolvimento de competências pelo estudante e o seu maior protagonismo neste processo exigem do docente uma crescente atualização profissional ${ }^{1}$, com o objetivo de fundamentar a formação técnica, científica, ético-humanista ${ }^{2,3}$, em conformidade com a realidade social contemporânea.., 5

Em decorrência disso, as circunstâncias profissionais suscitam ao docente desafios cotidianos alicerçados na grande responsabilidade que lhe é atribuída. ${ }^{6}$ Desse modo, é possível evidenciar que os aspectos que tecem a dinâmica dos meios universitários podem interferir diretamente na integridade da saúde mental e, por conseguinte, na prática docente..$^{?}$

Nesse cenário, identifica-se uma lógica baseada nas responsabilidades profissionais e sociais. Como consequência, tem-se uma maior carga horária de dedicação ao trabalho, a qual se torna majoritariamente supervalorizada em detrimento às questões pessoais, com a finalidade de consolidar o máximo de sua eficiência. Assim, o docente se expõe a um processo contínuo e intenso de desgaste emocional, que pode repercutir nas dimensões da Síndrome de Burnout (SB).., 9

Em contrapartida, outro conceito tem sido discutido no ambiente de trabalho do mundo atual: o engagement. Este corresponde à capacidade que o indivíduo tem de extrair pontos positivos da realidade em que é exposto no trabalho. ${ }^{210}$ Associado a um constructo motivacional, cujas esferas abarcam o vigor, a dedicação e a eficácia, o engagement tem se categorizado como uma estratégia primordial das grandes empresas e das universidades para valorizar a atuação do profissional, evitando o estresse crônico e os quadros de esgotamento e desumanização. ${ }^{11}$
Portanto, levando em consideração que os fatores atrelados ao engagement e à Síndrome de Burnout são constructos que permeiam o exercício docente em suas múltiplas habilidades, o objetivo deste estudo é evidenciar pela revisão de literatura científica como se dá o engagement e Síndrome de Burnout na docência no ensino superior na área da saúde.

\section{Método}

Trata-se de revisão integrativa da literatura, realizada em seis etapas. $\stackrel{12}{ }$ Para tanto, tem-se como questão de pesquisa "o que versa a literatura científica acerca do engagement e da Síndrome de Burnout na docência no ensino superior na área da saúde?"

A busca pelos estudos foi realizada em três bases de dados: Biblioteca Virtual em Saúde (BVS), Web of Science e Science Direct. Estabeleceram-se como critérios de inclusão: artigos correspondentes à pergunta de pesquisa, artigos originais nos idiomas português, inglês e espanhol, independente do recorte temporal e trabalhos disponíveis na íntegra. Em contrapartida, os critérios de exclusão referem-se a artigos não relacionados ao tema e estudos não disponíveis para a leitura na íntegra, optou-se por não determinar o intervalo temporal das publicações devido ao número reduzido de estudos. Utilizados como descritores relacionados à Síndrome de Burnout "faculty", "burnout" e "higher education", totalizando 19 estudos na BVS e Web of Science e 293 na Science Direct. Para a pesquisa referente ao engagement, foram empregados os seguintes descritores: "faculty", "work engagement" e "higher education", resultando em 5 artigos na Web of Science, 1 na BVS e 35 na Science Direct.

A partir da leitura criteriosa dos resumos, seguida da leitura na íntegra dos artigos, foram selecionados 6 artigos para o estudo. (Figura 1) 
Figura 1. Fluxograma de identificação e seleção dos artigos para revisão integrativa

Bases de dados: BVS, Science Direct e Web of Science
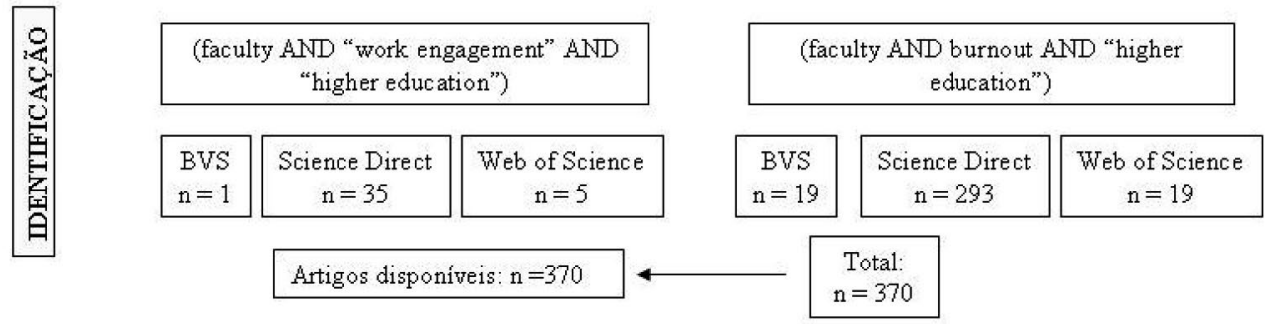

绶

Estudos selecionados na íntegra após remoção das duplicações: $\mathrm{n}=88$

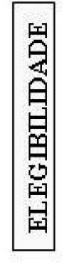

Critérios de inclusão: estudos originais nos idiomas inglês, português e espanhol,

independente do recorte temporal $n=87$

\begin{tabular}{|c|c|}
\hline $\begin{array}{l}\text { Estudos que abordaram a } \\
\text { temática Engagement na } \\
\text { docência no ensino superior na } \\
\text { área da saúde } \mathrm{n}=18 \text {. }\end{array}$ & $\begin{array}{l}\text { Estudos que abordaram a temática } \\
\text { sindrome de Bumout na docência no } \\
\text { ensino superior na área da saúde } \mathrm{n}= \\
63 \text {. }\end{array}$ \\
\hline
\end{tabular}

Estudos incluidos na revisão: 6

Fonte: Os autores (2021).

Os estudos incluídos na revisão foram relacionados em um quadro sinóptico, de acordo com as seguintes variáveis: ano de publicação, título, autores, periódico, tipo de estudo e resultados, que serão apresentados a seguir.

\section{Resultados}

A organização das informações em um quadro sinóptico permitiu a análise crítica dos estudos primários selecionados, levando em consideração a temática da pesquisa: engagement e Síndrome de Burnout na docência no ensino superior na área da saúde (QUADRO 1). 


\begin{tabular}{|c|c|c|c|c|c|}
\hline ANO & TÍTULO & AUTORES & PERIÓDICO & TIPO DE ESTUDO & RESULTADOS \\
\hline 2017 & $\begin{array}{l}\text { College nursing faculty job } \\
\text { satisfaction and retention: } \\
\text { A national perspective. }\end{array}$ & $\begin{array}{l}\text { Lee, P. Miller, MT. } \\
\text { Kippernbrock, } \\
\text { TA. Rosen, C. } \\
\text { Emory, J. }\end{array}$ & $\begin{array}{l}\text { Journal of } \\
\text { Professional } \\
\text { Nursing. }\end{array}$ & $\begin{array}{c}\text { Estudo } \\
\text { quantitativo. }\end{array}$ & $\begin{array}{l}\text { Confirmam uma } \\
\text { diversidade de } \\
\text { variáveis modificáveis } \\
\text { que são vistas como } \\
\text { importantes para } \\
\text { dedicação e } \\
\text { permanência na } \\
\text { carreira como } \\
\text { professor no curso de } \\
\text { enfermagem, } \\
\text { principalmente em } \\
\text { relação a assumir um } \\
\text { cargo de liderança. }\end{array}$ \\
\hline 2017 & $\begin{array}{c}\text { Avaliação da síndrome de } \\
\text { Burnout em professores } \\
\text { universitários. }\end{array}$ & $\begin{array}{l}\text { Rosana Leal do } \\
\text { Prado, Mariana } \\
\text { Esperendi } \\
\text { Bastianini, } \\
\text { Matheus } \\
\text { Zanelato } \\
\text { Cavalleri, Sandra } \\
\text { Fogaça Rosa } \\
\text { Ribeiro, Eliane } \\
\text { Cristina Gava Pizi } \\
\text { e Juliane Avansini } \\
\text { Marsicano. }\end{array}$ & $\begin{array}{l}\text { Revista de } \\
\text { ABENO. }\end{array}$ & $\begin{array}{l}\text { Estudo } \\
\text { exploratório, } \\
\text { descritivo e } \\
\text { transversal. }\end{array}$ & $\begin{array}{c}\text { As variáveis que } \\
\text { demonstraram } \\
\text { significância estatística } \\
\text { na correlação com os } \\
\text { níveis de Burnout } \\
\text { foram a quantidade de } \\
\text { cursos que o docente } \\
\text { leciona e a quantidade } \\
\text { de alunos com os quais } \\
\text { tem contato } \\
\text { diariamente e a idade e } \\
\text { o tempo de trabalho. }\end{array}$ \\
\hline 2013 & $\begin{array}{l}\text { Stressors of dual- } \\
\text { qualification nursing } \\
\text { teachers in the ChengDu - } \\
\text { ChongQing economic zone } \\
\text { of China - A qualitative } \\
\text { study. }\end{array}$ & $\begin{array}{c}\text { Wu Y. Liu, H. He, } \\
\text { H. }\end{array}$ & $\begin{array}{l}\text { Journal of } \\
\text { Professional } \\
\text { Nursing. }\end{array}$ & $\begin{array}{c}\text { Estudo } \\
\text { qualitativo. }\end{array}$ & $\begin{array}{c}\text { A carga de trabalho } \\
\text { pesada, segurança } \\
\text { pessoal, remuneração } \\
\text { inadequada, } \\
\text { sobrecarga de papéis e } \\
\text { ambiente de trabalho } \\
\text { precário são fatores } \\
\text { estressores. }\end{array}$ \\
\hline 2013 & $\begin{array}{l}\text { Prevalência da Síndrome } \\
\text { de Burnout em uma } \\
\text { amostra de professores } \\
\text { universitários brasileiros. }\end{array}$ & $\begin{array}{l}\text { Ludmila da Silva } \\
\text { Tavares Costa, } \\
\text { Pedro Rafael Gil- } \\
\text { Monte, Rosana } \\
\text { de Fátima } \\
\text { Possobon e } \\
\text { Glaucia Maria } \\
\text { Bovi Ambrosano. }\end{array}$ & $\begin{array}{l}\text { Psicologia: } \\
\text { Reflexão e } \\
\text { Crítica. }\end{array}$ & $\begin{array}{l}\text { Estudo descritivo, } \\
\text { exploratório, } \\
\text { qualitativo. }\end{array}$ & $\begin{array}{c}\text { Profissionais que } \\
\text { apresentam baixa } \\
\text { Ilusão pelo trabalho, } \\
\text { altos níveis de } \\
\text { desgaste psíquico e } \\
\text { indolência } \\
\text { acompanhados de } \\
\text { sentimento de culpa, } \\
\text { podem ser } \\
\text { considerados como } \\
\text { casos de síndrome de } \\
\text { Burnout (SB) pela } \\
\text { legislação brasileira. } \\
\text { Os resultados } \\
\text { mostraram que 11,2\% } \\
\text { dos professores } \\
\text { apresentaram perfil } 1 \text { e } \\
\text { 3\% perfil } 2 \text { da SB. }\end{array}$ \\
\hline 2012 & $\begin{array}{l}\text { Síndrome de Burnout em } \\
\text { Médicos Docentes de uma } \\
\text { Instituição Pública. }\end{array}$ & $\begin{array}{l}\text { Vera Lúcia Gama } \\
\text { de Mendonça, } \\
\text { Jorge Artur } \\
\text { Peçanha de } \\
\text { Miranda Coelho } \\
\text { e Mário Jorge } \\
\text { Júca. }\end{array}$ & $\begin{array}{c}\text { Psicologia em } \\
\text { Pesquisa - UFJF. }\end{array}$ & $\begin{array}{c}\text { Estudo } \\
\text { correlacional e } \\
\text { de comparação. }\end{array}$ & $\begin{array}{c}\text { Quanto maior a } \\
\text { demanda de trabalho, } \\
\text { maior a exaustão } \\
\text { emocional e } \\
\text { despersonificação e } \\
\text { menor a realização } \\
\text { profissional. Quanto } \\
\text { menor o controle e } \\
\text { apoio social, maior o } \\
\text { grau de exaustão } \\
\text { emocional e } \\
\text { despersonificação na } \\
\text { medida em que quanto } \\
\text { maior o controle. }\end{array}$ \\
\hline 2011 & $\begin{array}{l}\text { Analysis of Head of } \\
\text { Departments Leadership } \\
\text { Styles: Implication for } \\
\text { Improving Research } \\
\text { University Management } \\
\text { Practices. }\end{array}$ & $\begin{array}{l}\text { Zaidatol } \\
\text { Akmaliah Lope } \\
\text { Pihie, Amir } \\
\text { Sadeghi e } \\
\text { Habibah Elias. }\end{array}$ & $\begin{array}{c}\text { Procedia - Social } \\
\text { and Behavioral } \\
\text { Sciences. }\end{array}$ & $\begin{array}{l}\text { Estudo descritivo } \\
\text { exploratório. }\end{array}$ & $\begin{array}{c}\text { A utilização da } \\
\text { liderança } \\
\text { transformacional } \\
\text { aumentou a satisfação } \\
\text { dos docentes. }\end{array}$ \\
\hline
\end{tabular}

Fonte: Os autores (2021). 
Tecendo um panorama sobre os estudos selecionados, conforme o ano de publicação, 33,3\% dos artigos foram desenvolvidos em 2017, 33\% em 2013 seguidos por um (17\%) em 2012 e em 2011, respectivamente.

Além disso, a discussão do tema é evidenciada em diversos países, circunstância consonante com este estudo, uma vez que dos seis trabalhos selecionados para análise, 50\% foi redigida no território brasileiro, 17\% das publicações nos Estados Unidos da América, China e Malásia, consecutivamente.

Outro aspecto relevante relaciona-se à presença dos conceitos centrais desse estudo nas diversas revistas científicas internacionais de múltiplas especialidades da área da saúde, haja vista que $50 \%$ das pesquisas foram publicadas em periódicos relacionados à Enfermagem, enquanto os demais foram publicados em revistas das áreas de Psicologia e Ciências Comportamentais. Este cenário se articula com a formação universitária, bem como a titulação acadêmica dos pesquisadores, visto que o número de enfermeiros foi predominante entre os autores, correspondendo aproximadamente a $35 \%$. Em seguida, tem-se os odontólogos (26\%), acompanhados pelos psicólogos (17\%) e médicos (13\%). Ademais, o restante das publicações incorporou engenheiro (4\%) e educador (4\%) como autores dos estudos analisados.

Em relação ao método empregado nos estudos, nota-se que a abordagem quantitativa foi dominante - presente em $83 \%$ dos artigos selecionados. Destacam-se entre as pesquisas quantitativas, os estudos correlacionais. Em paralelo, salienta-se que o artigo excedente (17\%) empregou a aplicação de uma escala, embora sua abordagem seja qualitativa. Quanto à amostra ou participantes dos estudos, $17 \%$ explicitaram especificamente profissionais médicos, enquanto $17 \%$ eram docentes enfermeiros. Complementarmente, $66 \%$ dos artigos expuseram de forma geral professores dos cursos na área da saúde como participantes do estudo.

Ainda, em $67 \%$ dos estudos a temática Síndrome de Burnout e/ou fatores estressores para o docente da área da saúde teve um maior destaque, enquanto que $33 \%$ abordaram satisfação laboral e fatores que contribuem para a retenção de profissionais nas instituições de ensino, tais como a autonomia e a criação estratégias de enfrentamento (coping).
Cabe salientar que as particularidades sociais e culturais de cada sociedade também se configuram como fatores relevantes, no que se refere à satisfação laboral e é importante destacar a pertinência da temática, no que diz respeito aos países de publicação dos estudos selecionados, o que demonstra o singular espectro das diversidades culturais, sociais e políticas da prática docente.

$\mathrm{Na}$ análise acerca dos resultados dos trabalhos selecionados nesta pesquisa, quanto à SB tem-se com maior frequência as variáveis idade, sexo e atividades de extensão do docente. ${ }^{13-15}$

Quanto à variável idade, houve predominância de docentes na quarta ${ }^{13,15}$ e quinta ${ }^{14}$ décadas de vida, o que demonstra a íntima ligação entre o desenvolvimento da SB em docentes com idade menos avançada, pois tendem a apresentar maior exaustão profissional e maior despersonalização, que se justificam pela frustração de expectativas e maior sentimento de distância pessoal no ambiente de trabalho. $\frac{15}{}$ Deste modo, quanto menor o controle e apoio social que o docente tem, maior é o seu grau de exaustão emocional. ${ }^{14}$

Em relação ao sexo, encontra-se a predominância de docentes do sexo masculino. ${ }^{13-11}$ Nos homens, há destaque para a não realização profissional, vinculada às expectativas de sucesso, competição e desenvolvimento relacionadas ao seu papel. ${ }^{15} \mathrm{Em}$ contrapartida, a maior predominância de exaustão emocional foi relacionada ao sexo feminino, e este fato está vinculado à necessidade de acolhimento, cuidado e atenção aos discentes, assemelhando-se ao "papel materno", acrescida ainda das demandas pessoais das docentes. $\frac{15}{}$

A exaustão emocional também está relacionada à quantidade de cursos nos quais o docente leciona e as atividades de extensão, desde projetos, orientações de trabalhos até participações em núcleos e colegiados. Este sentimento é explicado devido a alta demanda de trabalho e o contato diário com um grande número de estudantes. ㄴ.15 $^{15}$ Logo, observa-se que, quanto maior a demanda, maior será a exaustão emocional, despersonalização e consequentemente, menor a realização profissional. ${ }^{14}$ 
Ainda sobre SB, constatou-se uma alta carga de trabalho, cerca de 40 a 50 horas semanais $\frac{13}{3}$ o que propicia a exaustão, levando a sentimentos de apatia e desânimo. $\frac{15}{} \mathrm{~A}$ excessiva demanda, além de favorecer a exaustão emocional, contribui para a despersonalização e menor realização profissional. ${ }^{14}$ Por conseguinte, compromete o bom relacionamento com os estudantes e a construção de um ambiente socializado. ${ }^{15}$ Ademais, no que tange à manifestação comportamental da SB, os trabalhos destacam a raiva ${ }^{13-14}$, bem como a frustração ${ }^{13-11}$ e a ansiedade ${ }^{13}$ como achados típicos da comorbidade em questão. Além disso, a constância do medo somada aos problemas de sono, principalmente, a insônia ${ }^{13}$ - também são reflexos esperados da síndrome.

Destaca-se que o processo desgastante da SB suscita o impacto negativo na qualidade do trabalho pedagógico, corroborando com o prejuízo na criatividade do docente e a minimização de sua motivação pelo trabalho. 14,17,18 Desse modo, entre as três dimensões da SB, a exaustão emocional se apresenta como a sua principal manifestação, seguida pela motivação emocional e, por fim, pela despersonalização. ${ }^{13-14} \mathrm{Em}$ estados mais avançados, é comum identificar o sentimento de culpa, que representa o resultado crônico de grandes e inflexíveis cobranças pessoais. ${ }^{13}$

Outrossim, tanto as características pessoais quanto as sociais, desencadeiam o estresse docente, o que influencia a avaliação de outrem, o desempenho em suas aulas, a relação com colegas de trabalho, conflito de papéis, falta de condições de trabalho e o apoio inadequado de amigos e familiares. Ainda, deve-se destacar que, quanto maior a inexperiência, em seu ambiente de trabalho, maior sua insegurança. $\frac{15}{5}$

No cenário universitário, pesquisa realizada com médicos docentes 14 enquadra o docente numa situação de constante crivo crítico, haja vista que ele necessita exercer habilidades complexas na área de ensino, pesquisa e extensão - condição correspondente com o exposto em estudo realizado com professores universitários. ${ }^{15}$ Contudo, tais demandas são frutos da natureza psicológica, a qual pode ser intensamente construída pelo excesso de responsabilidades laborais e pelo aumento do potencial autocrítico..$^{14-15}$
Para que haja a prevenção da SB, é necessário propiciar um ambiente de trabalho saudável, onde deve-se observar a amplificação de políticas, procedimentos e sistemas concebidos, de modo a fazer com que os colaboradores se tornem capazes de atingir os objetivos organizacionais e, concomitantemente, alcancem satisfação em sua ocupação profissional. $\frac{13,16}{16}$

\section{Discussão}

Percebe-se que significativas transformações ocorreram no papel docente, passando de profissional detentor do conhecimento no ensino tradicional, para aquele que busca novas estratégias de ensinagem, que é capaz de aprender novas tecnologias e assim inovar seus métodos em sala de aula, que tem o olhar no estudante para o desenvolvimento de competências. Todavia, diversos fatores podem impactar no grau de envolvimento docente, como a quantidade de cursos em que atua, alta carga de trabalho, sobrecarga de papeis, remuneração inadequada, dentre outros.

A docência no ensino superior na área da saúde se configura como uma categoria profissional intensamente exposta a estressores psicossociais, e conforme já abordado anteriormente, os constructos engagement e Síndrome de Burnout permeiam o exercício docente em suas múltiplas habilidades, sendo este último o foco da maioria dos estudos selecionados.

Os estudos relatam que, do ponto de vista conceitual, a Síndrome de Burnout (SB) é composta por três dimensões: a exaustão emocional (EE), a despersonalização (D) e a diminuição da realização pessoal (DRP). $\stackrel{13-15}{15}$ Tendo em vista que se trata de uma desordem na perspectiva social e psicológica, o agravamento da SB correlaciona-se a aspectos ocupacionais e ambientais, especialmente, em profissões que têm contato direto com pessoas, tais como professores, trabalhadores da área da saúde, assistentes sociais e agentes penitenciários, os quais tendem a apresentar maiores cargas emocionais, e cujas repercussões culminam numa exaustão emocional e despersonalização severas. Tais profissões são notoriamente contrastadas pelos estudos selecionados. 
A Síndrome de Burnout (SB) é definida como uma resposta aos estressores laborais, que podem ser definidos como agente químico ou biológico, sendo ele interno ou externo, que causa estresse ao organismo. Os estressores ${ }^{16}$ produzem uma resposta fisiológica, o que pode ocasionar danos ao organismo e resultar em sentimentos negativos, diminuindo o engajamento no trabalho.

A Síndrome de Burnout envolve atitudes e condutas negativas nas relações interpessoais e na organização, acarretando prejuízos aos elementos envolvidos. Em consonância, estudo realizado com professores universitários brasileiros ${ }^{13}$ elucida que a SB no contexto da docência categoriza-se como um fenômeno complexo e multidimensional, sendo altamente vinculado às experiências individuais do professor com o meio ocupacional.

A sobrecarga laboral se consolida como alicerce para fomentar - no próprio docente - distúrbios relacionais atrelados ao conflito e a ambiguidade de papéis, a relacionamentos conflituosos com os estudantes e com outros professores. Tais achados também são encontrados no trabalho realizado com docentes de sete hospitais-escola na China. ${ }^{17}$

Ao observar a associação do profissional de saúde com a docência, no Ensino Superior, deve-se considerar a necessidade de conciliar atividades de ensino, pesquisa e extensão, atendendo tanto às atividades administrativas como também as questões relacionadas à produção científica; sendo fatores desencadeadores ${ }^{14}$ de demanda de trabalho; logo, ocorrerá o aumento da possibilidade da incidência da SB neste público em questão.

De acordo com estudo realizado em uma amostra de professores universitários, no Brasil ${ }^{13}$, o indivíduo, ao desenvolver a SB, poderá apresentar dois distintos perfis: em um primeiro perfil pode apresentar sentimentos negativos vinculados ao exercício profissional, porém não incapacita o docente para tal atividade, em contrapartida, o segundo perfil, caracteriza-se por sintomas que compõem as três dimensões de Burnout, além da manifestação de sentimento de culpa.

Conforme estudo realizado na China ${ }^{16}$, quando o nível de estresse, advindo do exercício ocupacional é excedido, a capacidade de atender as demandas impostas pela docência se torna deficitária, originando os sentimentos de esgotamento profissional e, consequentemente, podendo ocasionar a SB. Entre os principais fatores determinantes para o desenvolvimento do estresse laboral, percebe-se a remuneração inadequada, a sobrecarga de papéis e a elevada carga horária do profissional, sendo esta última considerada um aspecto mais frequente e significativo, visto que produzirá danos tanto fisiológicos como psicológicos no docente.

Há, ainda, repercussão negativa no sistema educacional, no engajamento do profissional, no comportamento dos alunos e na qualidade das aulas expositivas, afetando, não somente o profissional docente, mas também o processo de ensino-aprendizagem. Constata-se também a clara influência dos aspectos culturais e do contexto laboral sobre os resultados de Burnout. 13,16

Em contrapartida para a satisfação laboral, para o engagement docente, o profissional precisa ter certa autonomia sobre o planejamento de suas horas, criar estratégias de enfrentamento (coping) ${ }^{14}$ para lidar com as dificuldades e possuir interação social com seus colegas e superiores, que pode ser considerado como fator protetivo para a SB.

Diante disso, a maior flexibilização de sua rotina, assim como a maior autonomia no trabalho são paradigmas fundamentais para a garantia da manutenção da motivação pessoal e do engagement do docente universitário. A liberdade de decidir sobre a forma como almeja trabalhar e conduzir a sua produtividade são aspectos singulares que permitem uma redução importante das altas demandas psicológicas no corpo docente. ${ }^{14}$

Paralelamente, a lógica da organização, na dinâmica laboral, atrelada aos diversos tipos de liderança das gerências instituídas se consolidam como fatores diretamente influenciadores na dimensão de engagement pessoal no trabalho. Em estudos ${ }^{17,18}$ realizados, com docentes, observou-se que há concordância sobre os efeitos significativos que o estilo de liderança de uma organização possui, diretamente, no maior engajamento de seus colaboradores. Ao relacionar os efeitos da satisfação ocupacional observa-se que há influência positiva na eficiência, produtividade, relações com os demais colaboradores, desempenho organizacional dos profissionais, minimização do absenteísmo e da rotatividade. 
O trabalho realizado com docentes do ensino superior na América do Norte ${ }^{17}$ frisa que há diversos contributivos para fortalecer o engagement docente com a prática de ensinar. Nesse âmbito, a retenção docente se enquadra como uma estratégia extremamente importante para a manutenção da qualidade do ensino acadêmico.2,17,18 Assim, quando há a presença de elementos estressores - destaque à falta de apoio orientador - tem-se, como resultado, uma menor retenção docente.

\section{Conclusão}

Ao desenvolver um cenário que favorece a saúde física, mental e emocional de seus colaboradores, a instituição de ensino perceberá as manifestações positivas palpáveis nos resultados produzidos, repercutindo no engajamento profissional docente, no comportamento dos estudantes, e na qualidade do processo de ensino e aprendizagem. Acredita-se que para que isso ocorra a organização deve fornecer um ambiente que proporcione apoio e facilidade no ensino, no serviço e na prática do corpo docente para que sejam alcançados os resultados esperados, além de atender a satisfação com o trabalho e incentivar para que ocorra a intenção de permanência na instituição de ensino. Portanto, depreende-se que, ao investir na saúde e na qualidade de vida do docente, consequentemente, há investimento na educação.

Percebe-se a necessidade de mais pesquisas que tragam a correlação entre burnout e engagement em docentes do ensino superior, em especial na área da saúde, uma vez que este estudo apontou a escassez de produções com esta temática.

\section{Contribuições dos autores}

Meyer LF, Gehr TE, Gonçalves LS, Santana FF, Mendes JO e Makuch DMV participaram da concepção, delineamento, busca e análise estatística dos dados da pesquisa, interpretação dos resultados e redação do artigo científico. Dal Ponte ARSL participou da busca e análise estatística dos dados da pesquisa. Zagonel IPS participou da concepção, delineamento, interpretação dos resultados, redação do artigo científico.

\section{Conflitos de interesses}

Nenhum conflito financeiro, legal ou político envolvendo terceiros (governo, empresas e fundações privadas, etc.) foi declarado para nenhum aspecto do trabalho submetido (incluindo, mas não se limitando a subvenções e financiamentos, participação em conselho consultivo, desenho de estudo, preparação de manuscrito, análise estatística, etc.).

\section{Referências}

1. Aleluia I, Boas LV, Brasil S, Zaidhaft S. Ser professor: quem sou eu e por quê? Rev Inter Educ Saúde. 2019;3(1):53-60. https://doi. org/10.17267/2594-7907ijhe.v3i1.2288

2. Nogueira MI. As mudanças na educação médica brasileira em perspectiva: reflexões sobre a emergência de um novo estilo de pensamento. Rev bras educ med. 2009;33(2):262-70. https://doi. org/10.1590/S0100-55022009000200014

3. Oliveira NA, Meirelles RMS, Cury GC, Alves LA. Mudanças curriculares no ensino médico brasileiro: um debate crucial no contexto do Promed. Revista Brasileira de Educação Médica. 2008;32(3):333-46. https://doi.org/10.1590/S0100$\underline{55022008000300008}$

4. Benevides-Pereira AM. Burnout: quando o trabalho ameaça o bem-estar do trabalhador. São Paulo: Casa do Psicólogo; 2002.

5. Freitas MAO, Cunha ICKO, Batista SHSS, Rossit RAS. Docência em saúde: percepções de egressos de um curso de especialização em Enfermagem. Interface. 2016;20(57):427-36. https://doi. org/10.1590/1807-57622015.0391

6. Araújo I, Esteves R. Engagement em docentes do ensino superior: uma abordagem exploratória. Enferméria Universitária. 2016;13(2):73-9. https://doi.org/10.1016/j.reu.2016.03.006

7. Treviso P, Costa BEP. The perception of professionals from the health area regarding their training as lecturers. Texto contexto - enferm. 2017;26(1). https://doi.org/10.1590/0104$\underline{07072017005020015}$

8. Carlotto MS. A síndrome de Burnout e o trabalho docente. Psicol. Estud. 2002;7(1):21-9. http://dx.doi.org/10.1590/S1413$\underline{73722002000100005}$

9. Castro FG, Zanelli JC. Síndrome de Burnout e projeto de ser [Internet]. Cad. psicol. soc. trab. 2007;10(2):17-33. Disponível em: http://pepsic.bvsalud.org/pdf/cpst/v10n2/v10n2a03.pdf

10. Andrade RS, Fernandes SRP, Bastos AVB. Bem-estar subjetivo e comprometimento com a carreira: examinando suas relações entre professores de ensino superior. Revpsico [Internet]. 2013;4(2):47-60. Disponível em: http://www.periodicos.ufc.br/ psicologiaufc/article/view/805 
11. Whitten DL. Mentoring and work engagement for female accounting, faculty members in higher education. Mentor Tutoring. 2017;24(5):365-82. http://dx.doi.org/10.1080/13611267. $\underline{2016.1275391}$

12. Mendes KS, Silveira RCCP, Galvão CM. Use of the bibliographic reference manager in the selection of primary studies in integrative reviews. Texto context - enferm. 2019;28(1):13. https:// doi.org/10.1590/1980-265X-TCE-2017-0204

13. Costa LST, Gil-Monte PR, Possobon RF, Ambrosano GMB. Prevalência da Síndrome de Burnout em uma Amostra de Professores Universitários Brasileiros. Psic. Reflex. e Crít., 2013, 26(4):636-642. http://dx.doi.org/10.1590/S010279722013000400003

14. Mendonça VLG, Coelho JAPM, Júca MJ. Síndrome de Burnout em Médicos Docentes de uma Instituição Pública. Psicol pesq. 2012;6(2):90-100. http://dx.doi.org/10.5327/Z198212472012000200002
15. Prado RL, Bastianini ME, Cavalleri MZ, Ribeiro SFR, Pizi ECG, Marsicano JA. Avaliação da síndrome de Burnout em professores universitários. Rev. ABENO. 2017;17(3):21-9. https://doi. org/10.30979/rev.abeno.v17i3.409

16. Wu Y, Liu H, He H. Stressors of dual-qualification nursing teachers in the ChengDu-ChongQing economic zone of China-A qualitative study. Nurse Educ Today. 2013;33:1496-500. https:// doi.org/10.1016/j.nedt.2013.05.003

17. Lee P, Miller MT, Kippenbrock TA, Rosen C, Emory J. College nursing faculty job satisfaction and retention: A national Perspective. J Prof Nurs. 2017;33(4):261-66. https://doi. org/10.1016/j.profnurs.2017.01.001

18. Pihie ZAL, Sadeghi A, Habibah E. Analysis of Head of Departments Leadership Styles: Implication for Improving Research University Management Practices. Procedia - Social and Behavioral Sciences. 2011;29:1081-90. https://doi.org/10.1016/j. sbspro.2011.11.341 